\section{P2.47 PATIENT INITIATED PARTNER THERAPY FOR CHLAMYDIA: ATTITUDE OF PHYSICIANS AND NURSES WORKING IN SEXUAL HEALTH CENTRES IN THE NETHERLANDS}

${ }^{1} \mathrm{M}$ Visser, ${ }^{1}$ Van Den Broek IV, ${ }^{1}$ Van Benthem Bh, ${ }^{2} \mathrm{Götz} \mathrm{Hm.} 1$ Centre For Infectious Diseases Control, National Institute for Public Health and The Environment, Bilthoven, The Netherlands; ${ }^{2}$ Municipal Public Health Service Rotterdam-Rijnmond, Rotterdam, The Netherlands

\subsection{6/sextrans-2017-053264.223}

Introduction Effective partner treatment (PT) is essential to interrupt transmission, prevent re-infections, and may reduce the prevalence of chlamydia. Dutch guidelines allow direct PT of current and most recent ex-partners of a chlamydia patient when they present for testing, but no prescriptions without contact with the partner. As part of a project concerning the potential of Patient Initiated PT (PIPT) for chlamydia (PICCUP; Patient Initiated Contact treatment for Chlamydia), we investigated attitudes towards PIPT among health staff at sexual health centres (SHC).

Methods An online anonymous questionnaire was sent to all 73 physicians and 248 nurses employed at 25 Dutch SHCs. The questionnaire was based on focus groups conducted with health staff, and included Likert-scale questions on opinions and attitudes towards PIPT. Descriptive analyses were performed.

Results The overall response rate was $36 \%$. In general, health staff was critical towards PIPT without counselling. $97 \%$ of respondents agreed that current steady partners should get treatment immediately, and $43 \%$ thought so for casual partners. However, a smaller proportion would give medication via the index patient: $51 \%$ for a steady and $8 \%$ for a casual partner. Respondents were more likely to apply PIPT if there was a high chance of infection but a small chance the partner would present for testing. Furthermore, checking allergies and contra-indications before antibiotic prescription was considered essential by $97 \%$. Most (60\%) preferred acquiring this information via direct contact with the partner; 30\% favoured telephone or internet. Hardly any differences were seen between answers of physicians or nurses.

Conclusion Physicians and nurses from SHCs find PT for chlamydia important, but their attitude towards PIPT is reluctant. They would consider PIPT for current steady partners or for partners who may not present for testing, preferably after some form of contact with the partner. For further development of PIPT strategies, involvement of the implementing health professionals is essential.

\section{P2.48 MOLECULAR SUBTYPING OF TREPONEMA PALLIDUM AND ASSOCIATED FACTORS OF SEROFAST STATUS IN EARLY SYPHILIS PATIENTS: IDENTIFIED NOVEL GENOTYPE AND CYTOKINE MARKER}

${ }^{1} \mathrm{RL}$ Zhang, ${ }^{25} \mathrm{QQ}$ Wang, ${ }^{2} \mathrm{JP}$ Zhang, ${ }^{1} \mathrm{LJ}$ Yang. ${ }^{1}$ Department of Dermatology, Wuxi Second Hospital, Nanjing Medical University, Wuxi, China; ${ }^{2}$ Institute of Dermatology, Chinese Academy of MedicalSciences, Nanjing, China

\subsection{6/sextrans-2017-053264.224}

Introduction Serofast remains a concern of clinicians and syphilis patients. No consensus has been established, however, that defines an effective treatment strategy and clarifies the pathogenesis.
Methods Between August 2011 and July 2015, eligible patients visiting the STD Clinics in ten prefectural-level cities in Jiangsu Province, China, were referred to participate in this study.A total of 517 patients with early syphilis were enrolled and treated in this study.

Results Twelve months after treatment, 79.3\% (410/517) of patients achieved serological cure, 20.1\% (104/517) were serofast, and $0.6 \%$ (3/517) were serological failures. Multivariate analysis demonstrated that older age ( $>40$ years) and lower baseline RPR titer $(\leq 1: 8)$ were associated with serofast status. We also identified $21 \mathrm{~T}$. pallidum molecular subtypes among early syphilis patients and detected a new subtype, 14i/a.Levels of chemerin were higher in the serum of serofast cases than serological cure cases, potentially indicating a novel cytokine marker for serofast in early syphilis patients after therapy.

Conclusion Our data suggested that older age ( $>40$ years) and lower baseline RPR titer $(\leq 1: 8)$ were associated with serofast status in patients with early syphilis. These results indicated that 14i/a type predicted an increasing risk of serofast status,as well as chemerin may be a novel cytokine marker of serofast outcome in early syphilis patients.

\section{P2.49 PREVALENCE AND MOLECULAR CHARACTERISATION OF HEPATITIS B VIRUS IN BLOOD DONORS IN BOTSWANA}

${ }^{1}$ Wonderful T Choga, ${ }^{2} \mathrm{M}$ Anderson, ${ }^{3} \mathrm{~S}$ Gaseitsiwe, ${ }^{4} \mathrm{~K}$ Kasvosve, ${ }^{2} \mathrm{~S}$ Moyo, ${ }^{5} \mathrm{R}$ Musonda, ${ }^{6} \mathrm{M}$ Essex. ${ }^{1}$ Botswana Harvard AIDS Institute Partnership, Gaborone - Botswana; ${ }^{2}$ Botswana Harvard AIDS Institute, Gaborone - Botswana; ${ }^{3}$ Harvard School of Public Health/Botswna Harvard AIDS Institute, Botson - Botswana; ${ }^{4}$ University of Botswana, Gaborone Botswana; ${ }^{5}$ Botswana Harvard Aids Institute, Botswana Harvard Aids Instituite - Botswana; ${ }^{6}$ Harvard T.Chan School of Public Health, Botson, USA

\subsection{6/sextrans-2017-053264.225}

Introduction Safe blood transfusions remain a challenge in Africa where sexually transmitted diseases (STIs) mainly hepatitis B virus (HBV), hepatitis C virus (HCV), syphilis and HIV are hyper-endemic. Amongst, HBV is ranked the $7^{\text {th }}$ leading cause of annual global deaths. HBV has up to 10 genotypes (A-J) and all show uniqueness in prognosis, response to therapy and geographic distribution. In Africa, 30\%. (A, D and E) of these genotypes have been identified circulating in different cohorts. Up to date, there is no data on the prevalence and molecular characterisation of $\mathrm{HBV}$ in blood donors in Botswana. The study aims to identify HBV genotypes circulating in Botswana blood donors and update its prevalence in comparison to other identified STIs in the cohort.

Methods A one-year cross-sectional study for consecutive hepatitis B surface antigen positive $(\mathrm{HBsAg}+)$ allogeneic blood donations confirmed using ELISA was done. HBV genome extraction was done using UltraSense DNA/RNA Kit followed by a $25 \mu \mathrm{l}$ polymerase chain reaction (PCR) reaction made of master-mix containing SuperScript Platinum III polymerase and two primers Core-F and Werle-AS covering $2.1 \mathrm{~kb}$ region (PreS2, PreS1, S and part of Pol region). Big Dye sequencing chemistry was performed on $82 \%$ (41/50) successful PCR products of which $87.8 \%$ were successfully sequenced and genotyped.

Results Sub-genotype A1 (48\%) serotype adw2 and D (52\%) serotype ayw2 were confirmed in the cohort. Escape mutations A120L and 130R in two genotype A samples were found associated with failure to vaccine and detection. Allogeneic donations at National Blood Transfusions Services in Botswana (NBTS) during study were 10798 . Prevalence of 
$\mathrm{HBsAg}^{+}$was $0.92 \%$. Co-infections confirmed were of Syphilis $1.72 \%, 1.54 \%$ for HIV and $0.43 \%$ for Hepatitis C. The total population's age was normally distributed with mean of 29 \pm 1.7 years and a range of 44 years.

Conclusion Predominant genotype amongst Botswana blood donors is D3. There is a major concern to address STIs in Botswana much work is being done on HIV but the results reflect a burden of all STIs.

\section{P2.50 DETECTION OF TREPONEMA PALLIDUM DNA IN THE BREAST MILK OF A FEMALE SYPHILIS PATIENT IN SHENZHEN, CHINA}

Yang Fan, Caiyu Mao, Wenli Zhang, Tang Fen, Wang Feng, Hongfu Chang, Guan Yang. Shenzhen Centre for Chronic Disease Control and Prevention, Shenzhen - China Popular Republic

\subsection{6/sextrans-2017-053264.226}

Introduction To investigate whether there is Treponema pal$\operatorname{lidum}$ (TP) DNA in the breast milk of female patients with syphilis and provide scientific evidence for breast-feeding for female syphilis patients after childbirth.

Methods A polymerase chain reaction (PCR) technique was used for the detection of TP DNA in the breast milk of female syphilis patients in Shenzhen, China.

Results An early syphilis patient after six months childbirth with hard chancre in the labia majora and secondary syphilitic eruption in the trunk and limbs had toluidine red unheated serum test (TRUST) positive with the titer of 1:128 and Treponema pallidum particle agglutination (TPPA) positive and had TP DNA detected in her breast milk by PCR technique. Her six-month-old daughter had TRUST positive with the titer of 1:256 and TPPA positive with secondary syphilitic eruption in the trunk and limbs. The mother syphilis patient received 3 weekly intramuscular injections of 2.4 million units of benzathine penicillin G (BPG) on both sides, once a week. After one weekly intramuscular injection of BPG, TP DNA wasn't detected in the breast milk of the female patient and remained negative after two weekly injection of BPG in the breast milk of the female patient. Ten cases of syphilis before and during pregnancy received BPG treatment in our hospital all had no TP DNA detected in their breast milk.

Conclusion Female early syphilis patients never received BPG treatment have TP DNA detected in their breast milk and are unable to breast-feed their babies temporarily. Female syphilis patients received BPG treatment have no TP DNA detected in their breast milk and can breast-feed their babies but need to be followed-up regularly.

\section{P2.51 CLINICAL AND SEROLOGICAL OUTCOMES AFTER RETREATMENT OF SYPHILIS SEROFAST PATIENTS IN GUANGDONG PROVINCE, CHINA}

${ }^{1} \mathrm{X}$ Zhang, ${ }^{2}$ Shahum Andrea, 'LG Yang, ${ }^{3}$ Seña Ac, ${ }^{1} \mathrm{YH}$ Xue, ${ }^{1} \mathrm{HP}$ Zheng, ${ }^{1} \mathrm{~B}$ Yang. ${ }^{1} G$ uangdong Provincial Dermatology Hospital and Centre for Skin Diseases and STD Control, Guangzhou - China Popular Republic; ${ }^{2}$ University of North Carolina at Chapel Hill, Institute for Global Health and Infectious Diseases, Guangzhou, USA; ${ }^{3}$ University of North Carolina at Chapel Hill, Institute for Global Health and Infectious Diseases, Chapel Hill, USA

10.1136/sextrans-2017-053264.227

Introduction A high proportion of syphilis patients (pts) remain serofast with persistent nontreponemal (NT) antibody titers after treatment. It remains unclear whether patients should undergo further monitoring, retreatment, or lumbar punctures (LP) for cerebrospinal fluid (CSF) analysis. We analysed serofast subjects from China for their clinical characteristics and outcomes after retreatment.

Methods From 2014-2016, we retrospectively analysed data from cohort of syphilis serofast pts evaluated in STI clinics in Guangdong province, China. Serofast status was defined by $<4$ fold decline in NT-titers at $\geq 6-12$ months after treatment or persistent NT-titers at $\geq 12-24$ months following $a \geq 4$ fold decline. All pts with syphilis (except neurosyphilis [NS]) were treated with benzathine penicillin G (BPG) 2.4 million units for 3 weekly doses, and a subset had CSF analysis as per China syphilis guidelines. Treponema pallidum (Tp) PCR testing was performed among pts with whole blood specimens for analysis.

Results We enrolled 133 serofast pts, with a median age of 33 (IQR 31\%-43\%); 75\% were female and 99\% were HIV-negative. The initial diagnosis in $14 \%$ cases was early syphilis and $86 \%$ had late syphilis; $89(68 \%)$ had baseline NT-titers in the range 1:1-1:8. All pts had 3 doses of BPG as initial therapy, of which $74(56 \%)$ received retreatment $(51 \%>3$ doses of BPG, 18\% non-BPG, 31\% BPG+non-BPG). 61 (82\%) of those with retreatment failed to show $\geq 4$ fold decline of NTtiters after 1 year. LPs were performed among 82 (62\%) pts with median of 2.3 years (3-60 months) since diagnosis; only $4(5 \%)$ had CSF abnormalities, of which one symptomatic patient met criteria for probable NS. Tp-PCR testing was performed in a third of serofast patients and all had negative results.

Conclusion Most serofast cases had an initial diagnosis of late syphilis and presented with low baseline NT-titers. Most pts remained serofast despite retreatment. Among serofast pts who underwent CSF analysis and/or Tp-PCR testing, our findings suggest that persisting $T p$ infection is unlikely in the absence of symptoms.

\section{P2.52 RECURRENT JARISCH-HERXHEIMER REACTION}

E Macias-Del-Toro, L Nuñez-Hipolito, J Lopez-Robles, JL Ramirez-Bellver, JM RevellesMartinez, L Fuertes-De-Vega, JL Diaz-Recuero, L Requena-Caballero. Dermatology Department, Fundacion Jimenez Diaz, Madrid, Spain

\subsection{6/sextrans-2017-053264.228}

Clinical case We present the case of a 32 year-old man, HIV positive in treatment, that presented to our hospital. He had erythematous papules scattered through his trunk, limbs (including palms and soles) and genitalia. He denied previous genital, oral or anal ulcer. His previous serological tests for syphilis were negative. We suspected a secondary syphilis and prescribed Benzatin G Penicillin $2.4 \mathrm{M}$ IU. The patient referred to the emergency room for a flu-like reaction and worsening of the skin lesions after the injection. We performed a biopsy that confirmed the clinical diagnosis of syphilis, and the serological test came out positive (RPR 64). After the second injection of penicillin, the patient experienced a second reaction, with fever and malaise. Before the third treatment we administered prednisone for three days, and he had no reaction. The Jarisch-Herxheimer Reaction (JHR) is well known since the Middle Ages, when it was associated with mercury ointments used for the treatment of syphilis. The first literary descriptions came from Jarisch in 1895 and Herxheimer seven years later. Since then it has been described 